Pacific

Journal of

Mathematics

\title{
VARIATIONAL CHARACTERIZATIONS OF THE TOTAL SCALAR CURVATURE AND EIGENVALUES OF THE LAPLACIAN
}

Seungsu Hwang, Jeongwook Chang And GabJin Yun 


\title{
VARIATIONAL CHARACTERIZATIONS OF THE TOTAL SCALAR CURVATURE AND EIGENVALUES OF THE LAPLACIAN
}

\author{
Seungsu Hwang, Jeongwook Chang and GabJin Yun
}

\begin{abstract}
For the dual operator $s_{g}^{\prime *}$ of the linearization $s_{g}^{\prime}$ of the scalar curvature function, it is well-known that if ker $s_{g}^{\prime *} \neq 0$, then $s_{g}$ is a nonnegative constant. Moreover, if the Ricci curvature does not vanish, then $s_{g} /(n-1)$ is an eigenvalue of the Laplacian of the metric $g$. In this work, we give some variational characterizations for the space ker $s_{g}^{\prime *}$. To accomplish this, we introduce a fourth-order elliptic differential operator $\mathscr{A}$ and a related geometric invariant $v$. We prove that $v$ vanishes if and only if $\operatorname{ker} s_{g}^{\prime *} \neq 0$, and if the first eigenvalue of the Laplace operator is large compared to its scalar curvature, then $v$ is positive and $\operatorname{ker} s_{g}^{\prime *}=0$. We calculate a lower bound for $v$ in the case of $\operatorname{ker} s_{g}^{\prime *}=0$. We also show that if there exists a function which is $\mathscr{A}$-superharmonic and the Ricci curvature has a lower bound, then the first nonzero eigenvalue of the Laplace operator has an upper bound.
\end{abstract}

\section{Introduction}

Let $M$ be a compact smooth $n$-manifold (without a boundary). The space of all Riemannian metrics, $M$, on $M$ is then open in the space of symmetric 2-tensors, $\varphi^{2}(M)$, for the compact-open topology or the $W^{k, p}$-topology, where $W^{k, p}$ denotes the Sobolev space. For a Riemannian metric $g$ and a symmetric 2-tensor $h$, the differential $s_{g}^{\prime}(h)$ of the scalar curvature at $g$ in the direction $h$ is given by

$$
s_{g}^{\prime}(h)=-\Delta_{g} \operatorname{tr}(h)+\delta_{g}\left(\delta_{g} h\right)-g\left(r_{g}, h\right),
$$

where $\Delta_{g}$ is the negative Laplacian of $g$, and $r_{g}$ and $\delta_{g}$ denote the Ricci curvature and divergence operator of $g$, respectively [Besse 1987]. In addition, the $L^{2}$-adjoint

Hwang was supported by the Basic Science Research Program through the National Research Foundation of Korea (NRF) funded by the Ministry of Education, Science and Technology (MEST) grant 2011-0005211, Chang by MEST grant 2011-0005235, and Yun by MEST grant 2011-0007465. Yun is the corresponding author.

MSC2010: 53C21.

Keywords: critical point equation, fourth-order elliptic operator, eigenvalue, Einstein metric, Laplace operator, scalar curvature, total scalar curvature. 
operator $s_{g}^{\prime *}$ of $s_{g}^{\prime}$ is given by

$$
s_{g}^{\prime *}(f)=D d f-\left(\Delta_{g} f\right) g-f r_{g},
$$

where $D d f$ denotes the Hessian of $f$ with respect to the metric $g$. Note that both $s_{g}^{\prime}$ and $s_{g}^{\prime *}$ are linear second-order differential operators.

In this paper, we consider the fourth-order elliptic differential operator $\mathscr{A}=$ $s_{g}^{\prime} \circ s_{g}^{\prime *}: C^{\infty}(M) \rightarrow C^{\infty}(M)$. The existence of homogeneous or nonhomogeneous solutions to $\mathscr{A}$ is closely related to the kernel space of $s_{g}^{\prime *}$. For example, Bourguignon [1975] and Fischer and Marsden [1974] proved that if $\operatorname{ker} s_{g}^{\prime *} \neq 0$, then either $(M, g)$ is Ricci-flat and ker $s_{g}^{\prime *}=\mathbb{R} \cdot 1$, or the scalar curvature is a strictly positive constant and $s_{g} /(n-1)$ is an eigenvalue of the Laplacian. In particular, combined with the Lichnerowicz-Obata theorem [Lichnerowicz 1958; Obata 1962; Berger et al. 1971], it follows that if $g$ is an Einstein metric with positive scalar curvature, then $\operatorname{ker} s_{g}^{\prime *}=0$ or $g$ is the standard round metric on the sphere.

On the other hand, if $\operatorname{ker} s_{g}^{*}=0$, then for any function $\eta \in C^{\infty}(M)$ there exists a unique function $u \in C^{\infty}(M)$ such that $\mathscr{A}(u)=\eta$ (Theorem 2.2). In fact, the condition ker $s_{g}^{\prime *}=0$ implies the injectivity of $s_{g}^{\prime *}$ and the surjectivity of $s_{g}^{\prime}$. In order to perform variational characterizations of the condition $\operatorname{ker} s_{g}^{\prime *} \neq 0$, we introduce a geometric invariant $v$ which is defined by

$$
v=\inf \left\{\int_{M} \varphi \mathscr{A} \varphi d v_{g}\right\}
$$

where the infimum is taken over all functions $\varphi \in H^{2}(M)=W^{2,2}(M)$ with $\int_{M} \varphi^{2}=1$. Here $H^{2}(M)=W^{2,2}(M)$ denotes the Sobolev space which is $L^{2}$ up to the second (weak) derivatives.

A basic result related to the invariant $v$ is the following.

Theorem A. The invariant $v$ vanishes if and only if $\operatorname{ker} s_{g}^{*}=0$.

For the case $\operatorname{ker} s_{g}^{\prime *}=0$, we give a lower bound on $v$ and its relationship to the first nonzero eigenvalue of the Laplacian. We also show that if the first eigenvalue is large compared to the scalar curvature, then $v$ is positive and $\operatorname{ker} s_{g}^{*}=0$. In addition, if $M$ is the product of two standard spheres of the same dimension, then $v$ is exactly equal to the dimension of the spheres.

Theorem B. Let $M=S^{m} \times S^{m}(m \geq 2)$ with the standard product metric. Then

$$
v=m=\frac{\operatorname{dim}(M)}{2} .
$$

We also obtain upper bounds for the first nonzero eigenvalue of the Laplace operator when $\mathscr{A}$ satisfies a condition on evaluating functions. We say that a Riemannian manifold $(M, g)$ satisfies the $\mathscr{A}$-superharmonic condition if there exists 
a smooth function $\varphi$ such that $M_{\varphi}^{+} \neq \varnothing$ and $\mathscr{A} \varphi \leq 0$ on $M_{\varphi}^{+}$, and $\Delta \varphi=0$ on the boundary $\partial M_{\varphi}^{+}$of $M_{\varphi}^{+}$. Here $M_{\varphi}^{+}=\{x \in M: \varphi(x)>0\}$. For example, if $M$ is the standard sphere, then the first eigenfunction of the Laplacian satisfies these conditions. In general, any compact Riemannian manifold $(M, g)$ with positive scalar curvature and $\operatorname{ker} s_{g}^{* *} \neq 0$ satisfies the $\mathscr{A}$-superharmonic condition.

One of our main results is the following.

Theorem C. Let $\left(M^{n}, g\right)$ be a compact $n$-dimensional Riemannian manifold with a positive constant scalar curvature $s_{g}$. Suppose that $(M, g)$ satisfies the Asuperharmonic condition. If $\mathrm{Ric}_{g} \geq k \geq 0$, then the first nonzero eigenvalue $\lambda_{1}$ of the Laplacian satisfies

$$
\lambda_{1} \leq \frac{2 s_{g}-k+\sqrt{k^{2}-4 k s_{g}+4 s_{g}^{2} / n}}{2(n-1)} .
$$

Inequality (1-3) is sharp since the equality holds for the standard sphere. In performing analysis with the operator $\mathscr{A}$, the main difficulty is that we cannot apply the theory of second-order elliptic partial differential equations directly since $\mathscr{A}$ is a fourth-order differential operator.

The kernel space of $s_{g}^{\prime *}$ plays an important role in the critical point equation arising from the total scalar curvature functional. Let $\mu_{1}$ be the set of all smooth Riemannian metrics of unit volume on $M$, and let $\mathscr{b} \subset M_{1}$ be the set of all smooth Riemannian metrics on $M$ with constant scalar curvature, i.e.,

$$
\mathscr{C}=\left\{g \in M_{1}: s_{g}=\text { constant }\right\} .
$$

The total scalar curvature $\mathscr{S}: \mu_{1} \rightarrow \mathbb{R}$ is defined as

$$
\mathscr{S}(g)=\int_{M} s_{g} d v_{g}
$$

It is well-known that the total scalar curvature functional $\mathscr{S}$ restricted to $\mathscr{b}$ will be critical at $g$ if and only if there is a function $f$ with $\int_{M} f=0$ such that

$$
z_{g}=s_{g}^{\prime *}(f),
$$

where $z_{g}$ is the traceless Ricci tensor defined as $z_{g}=r_{g}-\left(s_{g} / n\right) g$. We call (1-4) the critical point equation (CPE). Note that if $f=0$, it follows from (1-4) that $z_{g}=0$, and thus $g$ is an Einstein metric. However, the existence of a nonzero solution is a very strong condition. The only known case with a nonzero solution is that of a standard sphere, and it has been conjectured that this is the only possible case [Besse 1987]. Namely, it is believed that if there exists a nonzero function $f$ satisfying the CPE, then $g$ must be an Einstein metric. We remark that a solution $(g, f)$ to the CPE is a nontrivial example of the $A$-superharmonic condition since $\mathscr{A} f=-\left|z_{g}\right|^{2}$ and $\Delta_{g} f=-\left(s_{g} /(n-1)\right) f$. 
Unless stated otherwise, we only consider Riemannian metrics on $M$ whose scalar curvatures are positive constants.

\section{Variational properties}

Let $(M, g)$ be a closed Riemannian $n$-manifold and $\delta$ be the adjoint operator of the differential $d$ with respect to the metric $g$. Unless explicitly stated, we will use $r$ rather than $r_{g}$ as the Ricci tensor of the metric $g$, and $s$ rather than $s_{g}$ as the scalar curvature. The following expressions are well-known definitions and identities: for a function $\varphi$ and any tensor $T$,

$\delta D d \varphi=-d \Delta \varphi-r(d \varphi, \cdot), \quad \delta d \varphi=-\Delta \varphi, \quad$ and $\quad \delta(\varphi T)=\varphi \delta T-T(d \varphi, \cdot)$.

Moreover, for any two functions $\varphi, \psi$,

$$
\psi\langle D d \varphi, r\rangle=-\delta(\psi r(d \varphi, \cdot))-r(d \varphi, d \psi) .
$$

Lemma 2.1. Let $\mathscr{A}=s_{g}^{\prime} \circ s_{g}^{\prime *}$ and assume the scalar curvature $s_{g}=s$ is constant. Then, for any function $\varphi$,

$$
\mathscr{A}(\varphi)=(n-1) \Delta^{2} \varphi+2 s \Delta \varphi-\langle D d \varphi, r\rangle+\varphi|r|^{2} .
$$

Proof. It follows directly from (1-2) that

$$
s_{g}^{\prime *}(\varphi)=D d \varphi-(\Delta \varphi) g-\varphi r
$$

and thus

$$
\mathscr{A}(\varphi)=s_{g}^{\prime} \circ s_{g}^{\prime *}(\varphi)=s_{g}^{\prime}(D d \varphi-(\Delta \varphi) g-\varphi r) .
$$

By (1-1), we have

$$
s_{g}^{\prime}(D d \varphi)=-\delta(r(d \varphi, \cdot))-\langle D d \varphi, r\rangle .
$$

Similarly, since $\delta g=0$ and $\delta r=-\frac{1}{2} d s=0$, we also obtain the following from (1-1): $s_{g}^{\prime}((\Delta \varphi) g)=(1-n) \Delta^{2} \varphi-s \Delta \varphi \quad$ and $\quad s_{g}^{\prime}(\varphi r)=-s \Delta \varphi+\delta(-r(d \varphi, \cdot))-\varphi|r|^{2}$.

Combining these two expressions, we obtain

$$
\mathscr{A}(\varphi)=(n-1) \Delta^{2} \varphi+2 s \Delta \varphi-\langle D d \varphi, r\rangle+\varphi|r|^{2} .
$$

Note that $\mathscr{A}$ is a fourth-order linear partial differential operator. The following theorem shows that $\mathscr{A}$ is elliptic and self-adjoint. We say that a fourth-order differential operator is elliptic if the symbol is injective.

Theorem 2.2. The operator $A$ is a self-adjoint, fourth-order elliptic linear operator. Furthermore, if $\operatorname{ker} s_{g}^{\prime *}=0$, then for any $\psi \in C^{\infty}(M)$ there exists a unique function $u \in C^{\infty}(M)$ such that $\psi=\mathscr{A}(u)$. 
Proof. We first show that $s_{g}^{\prime *}$ has injective symbol. Recall that for any $p \in M$ and any cotangent vector $t \in T_{p}^{*} M$, there is a linear map $\sigma_{t}\left(s_{g}^{\prime *}\right): T_{p} C^{\infty}(M) \rightarrow$ $T_{p} C^{\infty}\left(S^{2} M\right)$ called the symbol of $D=s_{g}^{\prime *}$, and the symbol of $D$ is called injective if $\sigma_{t}(D)$ is injective for all nonzero $t$. Note that for $t \in T^{*} M, \psi \in C^{\infty}(M)$,

$$
\sigma_{t}\left(s_{g}^{\prime *}\right) \cdot \psi=(-g(t, t) g+t \otimes t) \psi,
$$

which is clearly injective for $n>1$. Thus $s_{g}^{\prime *}$ is an operator of order 2 with injective symbol. By Lemma 4.4 of [Berger and Ebin 1969], $\mathscr{A}=s_{g}^{\prime} \circ s_{g}^{\prime *}$ is an elliptic operator of order 4 . It is clear from definition that $\mathscr{A}$ is self-adjoint.

Secondly, we show that $\mathscr{A}$ is surjective. Since $s_{g}^{\prime}$ is surjective, for any nontrivial $\psi \in C^{\infty}(M)$, there exists $\xi \in C^{\infty}\left(S^{2} M\right)$ such that $s_{g}^{\prime}(\xi)=\psi$. From the fact that $s_{g}$ is constant and the proof of Theorem 5.2 in the same reference, $C^{\infty}\left(S^{2} M\right)=$ $\operatorname{im} s_{g}^{\prime *} \oplus \operatorname{ker} s_{g}^{\prime}$. Thus, $\xi=\xi_{1}+\xi_{2}$ with $\xi_{1} \in \operatorname{im} s_{g}^{\prime *}$ and $\xi_{2} \in \operatorname{ker} s_{g}^{\prime}$. Therefore, for $\xi_{1}=s_{g}^{\prime *}(u)$, we have $\mathscr{A}(u)=\psi$.

Finally uniqueness comes from the assumption that $\operatorname{ker} s_{g}^{\prime *}=0$ since $\operatorname{ker} \mathscr{A}=$ $\operatorname{ker} s_{g}^{\prime *}$; clearly $\operatorname{ker} s_{g}^{\prime *} \subset \operatorname{ker} s_{g}^{\prime} \circ s_{g}^{\prime *}$, and $s_{g} \circ s_{g}^{\prime *}(u)=0$ implies

$$
0=\left(u, s_{g} \circ s_{g}^{\prime *}(u)\right)_{L^{2}}=\left(s_{g}^{\prime *}(u), s_{g}^{\prime *}(u)\right)_{L^{2}},
$$

where $(f, g)_{L^{2}}=\int_{M} f g d v_{g}$, and so $s_{g}^{*}(u)=0$.

Given a smooth compact $n$-dimensional Riemannian manifold $(M, g)$, we let $H^{2}(M)=W^{2,2}(M)$ be the Sobolev space defined as the completion of the space of smooth functions on $M$ with respect to the norm

$$
\|\varphi\|_{H^{2}(M)}^{2}=\int_{M}|D d \varphi|^{2} d v_{g}+\int_{M}|\nabla \varphi|^{2} d v_{g}+\int_{M} \varphi^{2} d v_{g} .
$$

To investigate the properties of operator $\mathscr{A}$ from the perspective of the calculus of variations, we define $E(\varphi)$ for any function $\varphi \in H^{2}(M)$ as

$$
E(\varphi)=\frac{1}{2} \int_{M}\left[(n-1)(\Delta \varphi)^{2}-2 s|d \varphi|^{2}+r(d \varphi, d \varphi)+\varphi^{2}|r|^{2}\right] .
$$

Since $\varphi\langle D d \varphi, r\rangle=\operatorname{div}(\varphi r(d \varphi, \cdot))-r(d \varphi, d \varphi)$, and thus

$$
\int_{M} \varphi\langle D d \varphi, r\rangle=-\int_{M} r(d \varphi, d \varphi),
$$

the Euler-Lagrange equation for the functional $E$ is exactly

$$
\mathscr{A}(\varphi)=(n-1) \Delta^{2} \varphi+2 s \Delta \varphi-\langle D d \varphi, r\rangle+\varphi|r|^{2}=0 .
$$


Note that if $\varphi=$ constant and $\mathscr{A}(\varphi)=0$, then $\varphi=0$ if the Ricci curvature $r$ does not identically vanish. Furthermore,

$$
E(\varphi)=\frac{1}{2} \int_{M} \varphi \mathscr{A}(\varphi)=\frac{1}{2} \int_{M}\left|s_{g}^{\prime *} \varphi\right|^{2} \geq 0
$$

for any function $\varphi$. In other words, $E$ is the energy of $\mathscr{A}$.

A simple direct observation is as follows.

Lemma 2.3. The kernel of $s_{g}^{*}$ vanishes if and only if $\operatorname{ker} \mathscr{A}=0$.

Proof. The proof follows from the fact that

$$
\int_{M}\left(s_{g}^{*} \varphi\right)^{2}=\int_{M} \varphi \mathscr{A}(\varphi)
$$

for any function $\varphi$. In fact, assume that $\operatorname{ker} \mathscr{A}=0$ and let $s_{g}^{\prime *} u=0$. Then $u$ realizes the infimum of $E(\varphi)$ among all smooth functions $C^{\infty}(M)$. That is, $u$ is a critical point for $E$, and thus $\mathscr{A}(u)=0$.

Example 2.4. Let $M$ be a round $n$-sphere $S^{n}$ with a standard round metric. Also, let $\varphi$ be the first nontrivial eigenfunction for the Laplacian so that

$$
\Delta \varphi=-n \varphi, \quad \int_{S^{n}}|d \varphi|^{2}=n \int_{S^{n}} \varphi^{2} .
$$

Since $r_{g}=(n-1) g$, it is easy to see that $E(\varphi)=0$. Thus the first eigenfunction $\varphi$ realizes the infimum of the functional $E$ and so

$$
\mathscr{A}(\varphi)=0 \quad \text { and } \quad \operatorname{ker} s_{g}^{\prime *} \neq 0 .
$$

On the other hand, consider $M=S^{n} \times S^{n+1}$ with the standard product metric. Then

$$
s_{g}=2 n^{2}, \quad\left|r_{g}\right|^{2}=n\left(2 n^{2}-n+1\right),
$$

and the first nonzero eigenvalue is given as

$$
\lambda_{1}(M)=\lambda_{1}\left(S^{n}\right)=n .
$$

Let $\varphi$ be the first eigenfunction corresponding to $\lambda_{1}(M)$ so that

$$
\Delta \varphi=-n \varphi, \quad r_{g}(d \varphi, d \varphi)=(n-1)|d \varphi|^{2} .
$$

Substituting (2-4) and (2-5) into (2-2), we obtain $E(\varphi)=0$. Therefore, we have $\mathscr{A}(\varphi)=0$, and thus $\operatorname{ker} s_{g}^{\prime *} \neq 0$.

Recall that $H^{2}(M)=W^{2,2}(M)$ is the Sobolev space consisting of functions that are $L^{2}$ up to the second (weak) derivative. Let

$$
\mathscr{W}=\left\{\varphi \in H^{2}(M): \int_{M} \varphi^{2}=1\right\}
$$


and define

$$
v=\inf \left\{\int_{M} \varphi \mathscr{A}(\varphi): \varphi \in \mathscr{W}\right\}
$$

Note that $v \geq 0$, and $\operatorname{ker} \mathscr{A} \neq 0$ implies $v=0$ by (2-3). The converse is also true.

Theorem 2.5. Suppose that $v=0$. Then $\operatorname{ker} \mathscr{A} \neq 0$.

Proof. Since $v=0$, there exists a sequence $\left(\varphi_{k}\right)$ of functions in $H^{2}(M)$ with $\int_{M} \varphi_{k}^{2}=1$ such that

$$
E\left(\varphi_{k}\right) \rightarrow 0 \quad \text { as } k \rightarrow \infty .
$$

We now claim that $\left(\varphi_{k}\right)$ is bounded in $H^{2}(M)$. On the contrary, suppose that the sequence $\left(\varphi_{k}\right)$ is unbounded in $H^{2}(M)$. Defining $\widetilde{\varphi}_{k}$ as

$$
\tilde{\varphi}_{k}=\frac{\varphi_{k}}{\left\|\varphi_{k}\right\|_{H^{2}(M)}}
$$

where $\left\|\varphi_{k}\right\|_{H^{2}(M)}$ denotes the Sobolev norm in $H^{2}(M)$, we have

$$
\left\|\widetilde{\varphi}_{k}\right\|_{H^{2}(M)}=1 \quad \text { and } \quad \int_{M} \tilde{\varphi}_{k}^{2} \rightarrow 0 \quad \text { as } k \rightarrow \infty .
$$

Furthermore, $E\left(\widetilde{\varphi}_{k}\right) \rightarrow 0$ as $k \rightarrow \infty$. Thus the rescaled sequence $\left(\widetilde{\varphi}_{k}\right)$ is bounded in $H^{2}(M)$ and so $\left(\widetilde{\varphi}_{k}\right)$ converges weakly to a function $\widetilde{\varphi}_{\infty} \in H^{2}(M)$. Applying the Rellich-Kondrakov embedding theorem $H^{2}(M) \subset H^{1}(M) \subset L^{2}(M), \widetilde{\varphi}_{k}$ converges strongly to $\tilde{\varphi}_{\infty}$ in $L^{2}$, and thus, there exists a subsequence, say $\left(\widetilde{\varphi}_{k}\right)$, that converges almost everywhere. However, since $\left\|\widetilde{\varphi}_{k}\right\|_{L^{2}(M)} \rightarrow 0$, the limit function $\tilde{\varphi}_{\infty}=0$, which is contradictory to the fact that $\|d \widetilde{\varphi}\|_{L^{2}(M)} \neq 0$ or $\|D d \widetilde{\varphi}\|_{L^{2}(M)} \neq 0$. Therefore, $\left(\varphi_{k}\right)$ is bounded, and so $\varphi_{k}$ converges weakly to a function $\varphi$ in $H^{2}(M)$. By the Rellich-Kondrakov embedding theorem again, it is easy to see that $\varphi_{k}$ converges strongly to $\varphi$ in $L^{2}(M)$, and thus, there exists a subsequence, say $\left(\varphi_{k}\right)$, that converges almost everywhere. Consequently, we have

$$
E(\varphi) \leq \liminf _{k \rightarrow \infty} E\left(\varphi_{k}\right)=0 .
$$

Hence since $E(\varphi)=0$ and $\int_{M} \varphi^{2}=1, \varphi$ is a nonconstant function and $\mathscr{A}(\varphi)=0$.

Corollary 2.6. The invariant $v$ vanishes if and only if $\operatorname{ker} s_{g}^{\prime *} \neq 0$ or $\operatorname{ker} \mathscr{A} \neq 0$.

Now we consider a special operator stemming from $\mathscr{A}$ that also plays a very important role in the kernel space of $s_{g}^{\prime *}$. For a function $\varphi$, define $P \varphi$ as

$$
P \varphi=(n-1) \Delta^{2} \varphi+2 s_{g} \Delta \varphi-\left\langle D d \varphi, r_{g}\right\rangle
$$

and define 


$$
\mu=\inf _{\substack{\varphi \in H^{2}(M) \\ \varphi \neq 0}} \frac{\int \varphi P \varphi}{\int \varphi^{2}} .
$$

Note that $\mu \leq 0$ since $P \varphi=0$ when $\varphi$ is a nonzero constant. Furthermore, it is easy to see that if $\mu=0$, then either $(M, g)$ is Ricci-flat or ker $\mathscr{A}=0$. In fact, if $u \in \operatorname{ker} \mathscr{A}$ and $r \neq 0$, then

$$
\int_{M} u P u=-\int_{M} u^{2}\left|r_{g}\right|^{2} \leq 0 .
$$

Since $\mu=0$ and $r_{g} \neq 0, u$ must be zero because $\int_{M} u^{2}\left|r_{g}\right|^{2}=0$. The following theorem shows that if $\operatorname{ker} \mathscr{A} \neq 0$, then $\mu$ must be nonpositive.

Theorem 2.7. Assume that $\operatorname{ker} \mathscr{A} \neq 0$ and $s=s_{g}$ is constant. Then

$$
-\max _{M}\left|r_{g}\right|^{2} \leq \mu \leq-\frac{s_{g}^{2}}{n} .
$$

Proof. Let $u \in \operatorname{ker} \mathscr{A}$ be a nonconstant function and $r$ be the Ricci tensor of the metric $g$. Since $s^{2} / n \leq|r|^{2}$, we have

$$
\mu \int_{M} u^{2} \leq \int_{M} u P u=-\int_{M} u^{2}|r|^{2} \leq-\frac{s^{2}}{n} \int_{M} u^{2}
$$

Thus

$$
\mu \leq-\frac{s^{2}}{n}
$$

On the other hand, it follows from Lemma 2.1 that

$\int_{M}\left(s_{g}^{\prime *} \varphi\right)^{2}=\int_{M} \varphi \mathscr{A}(\varphi)=\int_{M}\left\{(n-1)(\Delta \varphi)^{2}-2 s|d \varphi|^{2}-\varphi\langle D d \varphi, r\rangle+\varphi^{2}|r|^{2}\right\}$.

Thus,

$\int_{M}\left\{(n-1)(\Delta \varphi)^{2}-2 s|d \varphi|^{2}-\varphi\langle D d \varphi, r\rangle\right\} \geq-\int_{M} \varphi^{2}|r|^{2} \geq-\left(\max _{M}|r|^{2}\right) \int_{M} \varphi^{2}$.

Therefore, since

$$
\int_{M} \varphi P \varphi \geq-\left(\max _{M}|r|^{2}\right) \int_{M} \varphi^{2}
$$

for any function $\varphi$, we conclude that

$$
\mu \geq-\max _{M}|r|^{2}
$$


In view of Theorem 2.7, the invariant $\mu$ may designate a criteria for how close $g$ is to an Einstein metric. In fact, when $(M, g)$ is Einstein, it follows from Theorem 2.7 that, if $\operatorname{ker} \mathscr{A} \neq 0$,

$$
\mu=-\frac{s^{2}}{n} \text {. }
$$

In view of the operators $\mathscr{A}$ and $P$, for any real number $\alpha$, we introduce an elliptic fourth-order partial differential operator $\mathscr{A}_{\alpha}$ defined by

$$
\mathscr{A}_{\alpha}(\varphi)=(n-1) \Delta^{2} \varphi+2 s_{g} \Delta \varphi-\left\langle D d \varphi, r_{g}\right\rangle+(1-\alpha) \varphi\left|r_{g}\right|^{2},
$$

where $r_{g}$ is the Ricci tensor and $s_{g}$ is the scalar curvature, which is assumed to be a positive constant. Note that $\mathscr{A}_{0}=\mathscr{A}$ and $\mathscr{A}_{1}=P$.

Theorem 2.8. Assume that $\operatorname{ker} \mathscr{A}=0$ and $s=s_{g}$ is constant. Then there exists a positive real number $\alpha_{0}>0$ such that $\operatorname{ker} \mathscr{A}_{\alpha}=0$ for all $\alpha, 0 \leq \alpha \leq \alpha_{0}$.

Proof. For $0<\alpha<1$, let $u \in \operatorname{ker} \mathscr{A}_{\alpha}$ be a nontrivial function. Then

$$
\mathscr{A}(u)=\alpha u\left|r_{g}\right|^{2} \leq\left(\max _{M}\left|r_{g}\right|^{2}\right) \alpha u
$$

and so $v \leq\left(\max _{M}\left|r_{g}\right|^{2}\right) \alpha$. Since $\operatorname{ker} \mathscr{A}=0$, Corollary 2.6 states that $v>0$. Hence,

$$
0<\frac{v}{\max _{M}\left|r_{g}\right|^{2}} \leq \alpha .
$$

\section{Case of $v>0$}

In this section, we consider the case in which $v$ is positive, or, equivalently, $\operatorname{ker} \mathscr{A}=0$. We will investigate some necessary and sufficient conditions for $v$ to be positive and derive lower bounds on $v$.

Lemma 3.1. Assume $v>0$. Then

$$
\inf _{\varphi \in \mathscr{W}, \varphi \neq 1} \frac{E(\varphi)}{\|\varphi\|_{H^{2}(M)}}>0 .
$$

Here $\|\varphi\|_{H^{2}(M)}$ denotes the Sobolev norm in $H^{2}(M)$.

Proof. Suppose that

$$
\inf _{\varphi \in \mathscr{W}, \varphi \neq 1} \frac{E(\varphi)}{\|\varphi\|_{H^{2}(M)}}=0
$$

Then there exists a sequence $\left(\varphi_{k}\right)$ in $\mathcal{W}$ such that $\left\|\varphi_{k}\right\|_{L^{2}(M)}=1$ and

$$
\frac{E\left(\varphi_{k}\right)}{\left\|\varphi_{k}\right\|_{H^{2}(M)}} \rightarrow 0 \quad \text { as } k \rightarrow \infty
$$


Since $v>0$, we have $\left\|\varphi_{k}\right\|_{H^{2}(M)} \rightarrow \infty$ as $k \rightarrow \infty$. Defining $\widetilde{\varphi}_{k}$ as

$$
\tilde{\varphi}_{k}=\frac{\varphi_{k}}{\left\|\varphi_{k}\right\|_{H^{2}(M)}}
$$

we can obtain a contradiction, as in the proof of Theorem 2.5.

Theorem 3.2. Let $(M, g)$ be a compact Riemannian $n$-manifold with positive constant scalar curvature s. If $\operatorname{ker} \mathscr{A}=0$, then $v>0$ is contained in the spectrum of $\mathscr{A}$.

Proof. Recall that $\mathscr{W}=\left\{\varphi \in H^{2}(M): \int_{M} \varphi^{2}=1\right\}$. Theorem 2.5 and Lemma 3.1 imply that

$$
a:=\inf _{\varphi \in \mathscr{W}, \varphi \neq 1} \frac{E(\varphi)}{\|\varphi\|_{H^{2}(M)}}>0 .
$$

Then, for any function $\varphi \in \mathcal{W}$, we have $E(\varphi) \geq a\|\varphi\|_{H^{2}(M)}$, and thus,

$$
E(\varphi) \rightarrow \infty \quad \text { as }\|\varphi\|_{H^{2}(M)} \rightarrow \infty .
$$

In other words, the functional $E$ is coercive on $\mathcal{W}$.

On the other hand, let $\left(\varphi_{k}\right)$ be a sequence in $H^{2}(M)$ such that $\varphi_{k} \rightarrow \varphi$ weakly in $H^{2}(M)$. Then, according to the Rellich-Kondrakov theorem, $\varphi_{k} \rightarrow \varphi$ strongly in $L^{2}(M)$, and thus, a subsequence $\left(\varphi_{k}\right)$ converges almost everywhere. This shows that the subspace $\mathcal{W}$ is weakly closed in $H^{2}(M)$. Furthermore, since $M$ is compact, the subsequence $\left(\varphi_{k}\right)$ uniformly converges to $\varphi$, and we obtain

$$
E(\varphi) \leq \liminf _{k \rightarrow \infty} E\left(\varphi_{k}\right) .
$$

The functional $E$ is bounded below and attains its minimum in $H^{2}(M)$ [Struwe 1990]. Letting

$$
E(u)=\min \{E(\varphi): \varphi \in \mathcal{W}\},
$$

it is easy to see from the variational principle that

$$
\mathscr{A}(u)=v u .
$$

The properties of the operator $\mathscr{A}$ and the lower bound on $v$ are closely related to the first nonzero eigenvalue of the Laplacian. Let $\lambda$ be the first nonzero eigenvalue of the Laplace operator $\Delta$, which is characterized by

$$
\lambda=\inf \left\{\frac{\int_{M}|\nabla \varphi|^{2}}{\int_{M} \varphi^{2}}: \int_{M} \varphi=0\right\} .
$$

It follows from the characterization of the first nonzero eigenvalue that, for any function $\varphi$ with $\int_{M} \varphi=0$,

$$
\int_{M} \varphi^{2} \leq \frac{1}{\lambda} \int_{M}|d \varphi|^{2}
$$


Lemma 3.3. Let $\left(M^{n}, g\right)$ be a compact Riemannian n-manifold. Then, for any function $\varphi \in C^{\infty}(M)$,

$$
\int_{M}|d \varphi|^{2} \leq \frac{1}{\lambda} \int_{M}(\Delta \varphi)^{2} \leq \frac{n}{\lambda} \int_{M}|D d \varphi|^{2},
$$

where $\lambda$ is the first nonzero eigenvalue of the Laplacian.

Proof. It follows from integration by parts and the Cauchy-Schwarz inequality that

$$
\int_{M}|d \varphi|^{2} \leq \frac{1}{\lambda} \int_{M}(\Delta \varphi)^{2}
$$

The second inequality in (3-2) follows from the fact that $(\Delta \varphi)^{2} \leq n|D d \varphi|^{2}$.

Furthermore, for a function $\varphi$ with $\int_{M} \varphi=0$, we have

$$
\int_{M} \varphi^{2} \leq \frac{1}{\lambda^{2}} \int_{M}(\Delta \varphi)^{2} \quad \text { and } \quad \int_{M} \varphi^{2} \leq \frac{n}{\lambda^{2}} \int_{M}|D d \varphi|^{2} .
$$

A direct observation from the definition of $\mathscr{A}$ is the following theorem, which shows that if the first nonzero eigenvalue for the Laplacian is large compared to the sum of the scalar curvature and the norm of the Ricci tensor, then $v$ is positive.

Theorem 3.4. Let $\left(M^{n}, g\right)$ be a compact Riemannian $n$-manifold with positive constant scalar curvature s. If $(n-1) \lambda \geq 2 s+\max _{M}\left|r_{g}\right|$, then $v \geq s^{2} / n$, and thus $\operatorname{ker} \mathscr{A}=0$, or, equivalently, $\operatorname{ker} s_{g}^{\prime *}=0$.

Proof. Note that $\left|r_{g}\right|^{2} \geq s^{2} / n$. It follows from Lemma 3.3 that

$$
\int_{M}|d \varphi|^{2} \leq \frac{1}{\lambda} \int_{M}(\Delta \varphi)^{2}
$$

for any function $\varphi$. Thus, for any function $\varphi \in \mathcal{W}$,

$$
\begin{aligned}
E(\varphi) & =\frac{1}{2} \int_{M}(n-1)(\Delta \varphi)^{2}-2 s|d \varphi|^{2}+r_{g}(d \varphi, d \varphi)+\left|r_{g}\right|^{2} \varphi^{2} \\
& \geq \frac{1}{2}\left\{(n-1) \lambda-\left(2 s+\max \left|r_{g}\right|\right)\right\} \int_{M}|d \varphi|^{2}+\frac{s^{2}}{2 n} \int_{M} \varphi^{2} .
\end{aligned}
$$

Hence, $v \geq s^{2} / n$.

Remark 3.5. Assume $v>0$ for a compact Riemannian $n$-manifold $(M, g)$ with a positive constant scalar curvature. Then it follows from Theorem 3.2 that

$$
\mathscr{A}(u)=v u
$$

for some function $u \in \mathscr{W}$. In particular, we have

$$
\int_{M} u|r|^{2}=v \int_{M} u
$$


Since $\operatorname{ker} s_{g}^{*}=0$, by Theorem 2.2, there exists a unique function $\varphi \in C^{\infty}(M)$ such that $\mathscr{A}(\varphi)=u\left|r_{g}\right|^{2}$. Therefore,

$$
\frac{s^{2}}{n} \leq \int_{M} u^{2}\left|r_{g}\right|^{2}=\int_{M} u A \varphi=\int_{M} \varphi A u=v \int_{M} \varphi u \leq v\|\varphi\|_{L^{2}} .
$$

On the other hand, by the Cauchy-Schwarz inequality,

$$
\begin{aligned}
v\|\varphi\|_{L^{2}}^{2} & \leq \int_{M} \varphi \mathscr{A} \varphi=\int_{M} \varphi u\left|r_{g}\right|^{2} \leq\left(\int_{M} \varphi^{2}\left|r_{g}\right|^{2}\right)^{\frac{1}{2}}\left(\int_{M} u^{2}\left|r_{g}\right|^{2}\right)^{\frac{1}{2}} \\
& \leq\left(\max _{M}\left|r_{g}\right|\right)\|\varphi\|_{L^{2}} \sqrt{v\|\varphi\|_{L^{2}}} .
\end{aligned}
$$

Therefore, we have $v\|\varphi\|_{L^{2}} \leq \max _{M}\left|r_{g}\right|^{2}$, and so

$$
\frac{s^{2}}{n} \leq v\|\varphi\|_{L^{2}} \leq \max _{M}\left|r_{g}\right|^{2},
$$

where $\varphi$ is the function satisfying $\mathscr{A}(\varphi)=u\left|r_{g}\right|^{2}$.

Theorem 3.6. Let $M=S^{m} \times S^{m}(m \geq 2)$ with the standard product metric. Then

$$
v=m=\frac{\operatorname{dim}(M)}{2} \text {. }
$$

Proof. First, we will examine the case $m=2$ since key ingredients of the proof are contained in this setting. The cases of $m \geq 3$ will then be briefly explained.

For $M^{4}=S^{2} \times S^{2}$ with the standard product metric $g$, we obviously have $s_{g}=\left|r_{g}\right|^{2}=4, \lambda=2$, and $r_{g}=g$. Thus, $\langle D d \varphi, r\rangle=\Delta \varphi$ for any function $\varphi$, and so

$$
\mathscr{A}(\varphi)=3 \Delta^{2} \varphi+7 \Delta \varphi+4 \varphi .
$$

Let $u$ be a first eigenfunction of $S^{2}$ so that $\Delta u=-2 u, 2 \int_{M} u^{2}=\int_{M}|d u|^{2}$, and $r_{g}(d u, d u)=|d u|^{2}$. Therefore,

$$
\int_{M} u A(u)=\int_{M} 3(\Delta u)^{2}-7|d u|^{2}+4 u^{2}=2 \int_{M} u^{2} .
$$

Hence $v \leq 2$. To show the converse inequality $v \geq 2$, it is sufficient to prove that, for any $C^{\infty}$ function $\varphi$,

$$
F(\varphi):=\int_{M}\left[3(\Delta \varphi)^{2}-7|d \varphi|^{2}+2 \varphi^{2}\right] \geq 0 .
$$

First, note that

$$
F(\varphi)=\int_{M}(\Delta \varphi+2 \varphi)(3 \Delta \varphi+\varphi) .
$$

It follows from Lemma 3.3 that

$$
2 \int_{M}|d \varphi|^{2} \leq \int_{M}(\Delta \varphi)^{2}
$$


Thus, from the monotonicity of eigenvalues, it follows that, for any function $\varphi$ that vanishes on the smooth boundary $\partial D$ of a domain $D \subset M$, we have

$$
2 \int_{D}|d \varphi|^{2} \leq \int_{D}(\Delta \varphi)^{2} .
$$

Assume for a moment that 0 is a regular value of $\varphi$. Let $D_{1}$ be a region on $M$ such that

$$
\Delta \varphi+2 \varphi \leq 0 \quad \text { and } \quad \Delta \varphi+\frac{1}{3} \varphi \geq 0,
$$

and $D_{2}$ be a region such that

$$
\Delta \varphi+2 \varphi \geq 0 \quad \text { and } \quad \Delta \varphi+\frac{1}{3} \varphi \leq 0 .
$$

Note that $\varphi \leq 0$ on region $D_{1}$, and $\varphi \geq 0$ on region $D_{2}$. Thus, $\partial D_{1}=\partial D_{2}$. On region $D_{1}$, we have

$$
0<-\frac{1}{3} \varphi \leq \Delta \varphi \leq-2 \varphi .
$$

Multiplying (3-4) by $\varphi$ and integrating over $D_{1}$, we obtain

$$
-2 \int_{D_{1}} \varphi^{2} \leq \int_{D_{1}} \varphi \Delta \varphi \leq-\frac{1}{3} \int_{D_{1}} \varphi^{2} .
$$

Since $\varphi=0$ on $\partial D_{1}$, we get

$$
-2 \int_{D_{1}} \varphi^{2} \leq-\int_{D_{1}}|d \varphi|^{2} \leq-\frac{1}{3} \int_{D_{1}} \varphi^{2} .
$$

Similarly, on region $D_{2}$, we have

$$
-2 \int_{D_{2}} \varphi^{2} \leq \int_{D_{2}} \varphi \Delta \varphi \leq-\frac{1}{3} \int_{D_{2}} \varphi^{2} .
$$

Let $D=D_{1} \cup D_{2}$. It follows from (3-5) and (3-6) that

$$
\frac{1}{3} \int_{D} \varphi^{2} \leq \int_{D}|d \varphi|^{2} \leq 2 \int_{D} \varphi^{2} .
$$

Note that on $M-D$, we have

$$
(\Delta \varphi+2 \varphi)(3 \Delta \varphi+\varphi) \geq 0 .
$$

Furthermore, since the function $\varphi$ vanishes on the boundary $\partial D$ of $D$, we can apply integration by parts and Green's identity. Thus, it follows from (3-3), (3-7), and 
(3-8) that

$$
\begin{aligned}
F(\varphi) & =\int_{D}(\Delta \varphi+2 \varphi)(3 \Delta \varphi+\varphi)+\int_{M-D}(\Delta \varphi+2 \varphi)(3 \Delta \varphi+\varphi) \\
& =3 \int_{D}\left((\Delta \varphi)^{2}-2|d \varphi|^{2}\right)+\int_{D}\left(2 \varphi^{2}-|d \varphi|^{2}\right)+\int_{M-D}(\Delta \varphi+2 \varphi)(3 \Delta \varphi+\varphi) \\
& \geq 0 .
\end{aligned}
$$

Now, assume that 0 is a critical value of $\varphi$. By Sard's theorem, for any positive real number $\epsilon>0$, there exists a real number $a,-\epsilon<a<0$, such that $a$ is a regular value of $\varphi$. Let $D_{1, a}$ be a region such that

$$
\Delta \varphi+2 \varphi \leq \frac{5}{3} a \text { and } \Delta \varphi+\frac{1}{3} \varphi \geq 0 .
$$

Note that $\varphi \leq a<0$ on region $D_{1, a}$, and $\varphi=a$ on the boundary $\partial D_{1, a}$. Multiplying (3-9) by $\varphi$ and integrating it over $D_{1, a}$, we obtain

$$
\frac{5}{3} a \int_{D_{1, a}} \varphi-a \int_{\partial D_{1, a}} \frac{\partial \varphi}{\partial n_{1}} \leq \int_{D_{1, a}}\left(2 \varphi^{2}-|d \varphi|^{2}\right)
$$

where $n_{1}$ is the outward-pointing unit normal vector field to $\partial D_{1, a}$. Next, let $D_{2, a}$ be a region such that

$$
\Delta \varphi+2 \varphi \geq-\frac{5}{3} a \quad \text { and } \quad \Delta \varphi+\frac{1}{3} \varphi \leq 0 .
$$

We may assume that $-a$ is also a regular value of $\varphi$. Note that $0<-a \leq \varphi$ on region $D_{2, a}$, and $\varphi=-a$ on the boundary $\partial D_{2, a}$. Multiplying (3-11) by $\varphi$ and integrating it over $D_{2, a}$, we obtain

$$
a \int_{\partial D_{2, a}} \frac{\partial \varphi}{\partial n_{2}}-\frac{5}{3} a \int_{D_{2, a}} \varphi \leq \int_{D_{2, a}}\left(2 \varphi^{2}-|d \varphi|^{2}\right)
$$

where $n_{2}$ is a unit normal vector field on $\partial D_{2, a}$. Decomposing $M$ into three regions, we can write

$$
\begin{array}{r}
F(\varphi)=3 \int_{D_{1, a}}\left[(\Delta \varphi)^{2}-2|d \varphi|^{2}\right]+\int_{D_{1, a}}\left(2 \varphi^{2}-|d \varphi|^{2}\right)+3 \int_{D_{2, a}}\left[(\Delta \varphi)^{2}-2|d \varphi|^{2}\right] \\
+\int_{D_{2, a}}\left(2 \varphi^{2}-|d \varphi|^{2}\right)+\int_{M-\left(D_{1, a} \cup D_{2, a}\right)}(\Delta \varphi+2 \varphi)(3 \Delta \varphi+\varphi) .
\end{array}
$$

Applying inequality (3-3) to $\varphi-a$, we have

$$
\int_{D_{1, a}}\left[(\Delta \varphi)^{2}-2|d \varphi|^{2}\right] \geq 0 \quad \text { and } \quad \int_{D_{2, a}}\left[(\Delta \varphi)^{2}-2|d \varphi|^{2}\right] \geq 0 .
$$


Thus, from (3-10) and (3-12), we obtain

$$
\begin{aligned}
F(\varphi) \geq \frac{5}{3}|a| \int_{D_{1, a} \cup D_{2, a}}|\varphi|-a \int_{\partial D_{1, a}} & \frac{\partial \varphi}{\partial n_{1}}+a \int_{\partial D_{2, a}} \frac{\partial \varphi}{\partial n_{2}} \\
& +\int_{M-\left(D_{1, a} \cup D_{2, a}\right)}(\Delta \varphi+2 \varphi)(3 \Delta \varphi+\varphi) .
\end{aligned}
$$

Since $\left|\partial \varphi / \partial n_{1}\right| \leq|d \varphi|$ and $\left|\partial \varphi / \partial n_{2}\right| \leq|d \varphi|$, the first three terms on the right-hand side tend to 0 as $\epsilon \rightarrow 0$. Finally, let $E_{1, a}$ be a region such that $\Delta \varphi+2 \varphi>\frac{5}{3} a$ and $\Delta \varphi+\frac{1}{3} \varphi \geq 0$, and $E_{2, a}$ be a region such that $\Delta \varphi+2 \varphi<-\frac{5}{3} a$ and $\Delta \varphi+\frac{1}{3} \varphi \leq 0$. Then we have

$$
\int_{M-\left(D_{1, a} \cup D_{2, a}\right)}(\Delta \varphi+2 \varphi)(3 \Delta \varphi+\varphi) \geq \frac{5}{3} a \int_{E_{1, a}}(3 \Delta \varphi+\varphi)-\frac{5}{3} a \int_{E_{2, a}}(3 \Delta \varphi+\varphi) .
$$

The right-hand side tends to 0 as $\epsilon \rightarrow 0$. Hence, $F(\varphi) \geq 0$.

In the general case, $M^{2 m}=S^{m} \times S^{m}$ when $m \geq 2$, it is easy to see that

$$
s_{g}=2 m(m-1), \quad\left|r_{g}\right|^{2}=2 m(m-1)^{2}, \quad r_{g}=(m-1) g, \quad \lambda=m .
$$

Thus,

$$
\begin{aligned}
\int_{M} \varphi \mathscr{A}(\varphi)=(2 m-1) \int_{M}\left[(\Delta \varphi)^{2}-m|d \varphi|^{2}\right] & \\
& \quad-\left(2 m^{2}-4 m+1\right) \int_{M}|d \varphi|^{2}+2 m(m-1)^{2} \int_{M} \varphi^{2} .
\end{aligned}
$$

Using a first eigenfunction $u$ of $S^{m}, \Delta u=-m u$, we can demonstrate that $v \leq m$. To show that $v \geq m$, it is sufficient to prove that, for any function $\varphi$,

$$
F(\varphi):=\int_{M}(\Delta \varphi+m \varphi)\left[(2 m-1) \Delta \varphi+\left(2 m^{2}-4 m+1\right) \varphi\right] \geq 0 .
$$

Note that

$$
m \int_{M}|d \varphi|^{2} \leq \int_{M}(\Delta \varphi)^{2} .
$$

An argument identical to that used in the case $S^{2} \times S^{2}$ shows that $F(\varphi) \geq 0$, and thus, $v=m$.

Remark 3.7. For the case of $M=S^{m} \times S^{m+k}$ with $k \geq 2$, the first nonzero eigenfunction of $S^{m}$ can be used to show that

$$
v \leq \min \left\{(m+k)(k-1)^{2}, m(k+1)^{2}\right\} .
$$

However, we do not know the exact value of $v$. 


\section{The first eigenvalue of the Laplacian}

As mentioned above, the first nonzero eigenvalue $\lambda=\lambda_{1}(M)$ of the Laplace operator for a Riemannian manifold $(M, g)$ is related to the operator $\mathcal{A}$. For example, if ker $\mathscr{A} \neq 0$ and $g$ is an Einstein metric with positive scalar curvature, then $\lambda=s /(n-1)$, from the results obtained in [Berger et al. 1971] and [Bourguignon 1975]. We shall now see that, if there is a nontrivial function on which the action of $\mathscr{A}$ is nonpositive where the function is positive, then the first nonzero eigenvalue of the Laplacian is bounded above, and vice versa. Recall that we assumed that the scalar curvature $s_{g}=s$ of a Riemannian manifold $(M, g)$ is always a positive constant.

For a function $\varphi$ on a smooth manifold $M$, let us define

$$
M_{\varphi}^{+}=\{x \in M: \varphi(x)>0\} .
$$

We say that a Riemannian manifold $(M, g)$ satisfies the $\mathscr{A}$-superharmonic condition if there exists a smooth function $\varphi$ such that

(i) $M_{\varphi}^{+} \neq \varnothing$ and $\mathscr{A} \varphi \leq 0$ on $M_{\varphi}^{+}$;

(ii) $\Delta \varphi=0$ on the boundary $\partial M_{\varphi}^{+}$of $M_{\varphi}^{+}$.

For example, if $M=S^{n}$ with the standard round metric $g_{0}$, and $\varphi$ is the first nonzero eigenfunction of the Laplacian, i.e., $\Delta \varphi=-n \varphi$, then $A \varphi=0$ and $\left(S^{n}, g_{0}\right)$ satisfies the $\mathscr{A}$-superharmonic condition. Furthermore, note that any eigenfunction of the Laplacian satisfies condition (ii). The following lemma shows that the $\mathscr{A}$-superharmonic condition is implied by $\operatorname{ker} \mathscr{A} \neq 0$.

Lemma 4.1. Let $\left(M^{n}, g\right)$ be a compact $n$-dimensional Riemannian manifold with a positive constant scalar curvature $s_{g}$. If $\operatorname{ker} \mathscr{A} \neq 0$, then $(M, g)$ satisfies the A-superharmonic condition.

Proof. By Lemma 2.3, $\operatorname{ker} \mathscr{A} \neq 0$ is equivalent to $\operatorname{ker} s_{g}^{\prime *} \neq 0$. Let $s_{g}^{\prime *} \varphi=0$ and $\varphi \neq 0$. Then

$$
D d \varphi-(\Delta \varphi) g-\varphi r_{g}=0 .
$$

In particular, taking the trace yields

$$
\Delta \varphi=-\frac{s_{g}}{n-1} \varphi,
$$

and so $M_{\varphi}^{+} \neq \varnothing$. Since $\mathscr{A} \varphi=0$, the function $\varphi$ satisfies conditions (i) and (ii) in the definition of the $\mathscr{A}$-superharmonic condition.

Theorem 4.2. Let $\left(M^{n}, g\right)$ be a compact $n$-dimensional Riemannian manifold with a positive constant scalar curvature $s_{g}$. Suppose that $(M, g)$ satisfies the 
A-superharmonic condition. If $\operatorname{Ric}_{g} \geq k \geq 0$, then the first nonzero eigenvalue $\lambda_{1}$ of the Laplacian satisfies

$$
\lambda_{1} \leq \frac{2 s_{g}-k+\sqrt{k^{2}-4 k s_{g}+4 s_{g}^{2} / n}}{2(n-1)} .
$$

Proof. Let $s_{g}=s$ and $\operatorname{Ric}_{g}=r_{g}=r$. In addition, let $\varphi$ be a smooth function satisfying $M_{\varphi}^{+} \neq \varnothing, \mathscr{A} \varphi \leq 0$ on $M_{\varphi}^{+}$and $\Delta \varphi=0$ on the boundary $\partial M_{\varphi}^{+}$. If $\varphi$ is a constant function, then $\varphi$ is a positive constant since $M_{\varphi}^{+} \neq \varnothing$. However, we have $0 \geq \mathscr{A} \varphi=\varphi|r|^{2}$, which is a contradiction. Thus, we may assume that $\varphi$ is a nonconstant function. By the above hypothesis, we have

$$
\int_{M_{\varphi}^{+}} \varphi \mathscr{A} \varphi \leq 0 \text {. }
$$

By the definition of $\mathscr{A}$ and integration by parts, together with the fact that $\Delta \varphi=0$ on $\partial M_{\varphi}^{+}$, we obtain

$$
\begin{aligned}
\int_{M_{\varphi}^{+}} \varphi A \varphi= & \int_{M_{\varphi}^{+}}(n-1)(\Delta \varphi)^{2}- \\
& \int_{\partial M_{\varphi}^{+}} \Delta \varphi \frac{\partial \varphi}{\partial v} \\
& +\int_{M_{\varphi}^{+}}\left[2 s \varphi \Delta \varphi+\varphi\langle D d \varphi, r\rangle+|r|^{2} \varphi^{2}\right] \\
\geq & \int_{M_{\varphi}^{+}}\left[(n-1)(\Delta \varphi)^{2}+(2 s-k) \varphi \Delta \varphi+\frac{s^{2}}{n} \varphi^{2}\right] .
\end{aligned}
$$

Note that

$$
(n-1)(\Delta \varphi)^{2}+(2 s-k) \varphi \Delta \varphi+\frac{s^{2}}{n} \varphi^{2}=((n-1) \Delta \varphi+\alpha \varphi)(\Delta \varphi+\beta \varphi),
$$

where

$$
\alpha=\frac{2 s-k+\sqrt{k^{2}-4 k s+4 s^{2} / n}}{2}, \quad \beta=\frac{2 s-k-\sqrt{k^{2}-4 k s+4 s^{2} / n}}{2(n-1)} .
$$

Observe that $k^{2}-4 k s+4 s^{2} / n>0$ if and only if either

$$
k<2\left(1-\sqrt{1-\frac{1}{n}}\right) s_{g} \quad \text { or } \quad k>2\left(1+\sqrt{1-\frac{1}{n}}\right) s_{g},
$$

and the first inequality always holds.

Claim. If

$$
\lambda_{1}>\frac{2 s-k+\sqrt{k^{2}-4 k s+4 s^{2} / n}}{2(n-1)}=\frac{\alpha}{n-1},
$$

then any subset $\Omega$ of $M_{\varphi}^{+}$with $C^{1}$ boundary on which $(n-1) \Delta \varphi+\alpha \varphi \geq 0$ and $\Delta \varphi+\beta \varphi \leq 0$ has a measure of zero. 
Proof. Suppose that a subset $\Omega$ of $M_{\varphi}^{+}$contains an open $n$-ball. Note that since $\Delta \varphi=\varphi=0$ on $\partial \Omega$, we can apply the Dirichlet principle on the first nonzero eigenvalue of the Laplacian. By monotonicity, we have

$$
\lambda_{1}=\lambda_{1}(M) \leq \lambda_{1}(\Omega) .
$$

Since $(n-1) \Delta \varphi+\alpha \varphi \geq 0$ and $\varphi>0$ on $\Omega$, we have

$$
\varphi \Delta \varphi \geq-\frac{\alpha}{n-1} \varphi^{2} .
$$

Integrating this over $\Omega$, we obtain

$$
\int_{\Omega}|d \varphi|^{2} \leq \frac{\alpha}{n-1} \int_{\Omega} \varphi^{2} \leq \frac{\alpha}{n-1} \cdot \frac{1}{\lambda_{1}(\Omega)} \int_{\Omega}|d \varphi|^{2} .
$$

Thus,

$$
1 \leq \frac{\alpha}{n-1} \cdot \frac{1}{\lambda_{1}(\Omega)}
$$

and so

$$
\lambda_{1} \leq \lambda_{1}(\Omega) \leq \frac{\alpha}{n-1},
$$

which contradicts (4-5). This completes the proof of the claim.

Now, suppose that $\lambda_{1}>\frac{\alpha}{n-1}$. Since $\alpha>(n-1) \beta$, it follows from (4-4) and the above claim that

$$
(n-1)(\Delta \varphi)^{2}+(2 s-k) \varphi \Delta \varphi+\frac{s^{2}}{n} \varphi^{2} \geq 0 \quad \text { a.e. on } M_{\varphi}^{+},
$$

which implies that $\int_{M_{\varphi}^{+}} \varphi A \varphi \geq 0$. Consequently, from (4-2), we have

$$
\int_{M_{\varphi}^{+}} \varphi A \varphi=0 .
$$

Thus, on the set $M_{\varphi}^{+}$, we have $\mathscr{A} \varphi=0$ and

$$
(n-1)(\Delta \varphi)^{2}+(2 s-k) \varphi \Delta \varphi+\frac{s^{2}}{n} \varphi^{2}=((n-1) \Delta \varphi+\alpha \varphi)(\Delta \varphi+\beta \varphi)=0
$$

by (4-3). Since $\alpha>(n-1) \beta$, either $(n-1) \Delta \varphi+\alpha \varphi=0$ or $\Delta \varphi+\beta \varphi=0$ on the entire set $M_{\varphi}^{+}$. Therefore, we obtain

$$
\lambda_{1} \leq \lambda_{1}\left(M_{\varphi}^{+}\right) \leq \max \left\{\frac{\alpha}{n-1}, \beta\right\}=\frac{\alpha}{n-1},
$$

which contradicts the assumption $\lambda_{1}>\frac{\alpha}{n-1}$. Hence,

$$
\lambda_{1}=\lambda_{1}(M) \leq \frac{\alpha}{n-1} .
$$

This completes the proof of Theorem 4.2. 
Remark 4.3. If $M=S^{n}$ with the standard round metric, then taking $k=n-1$, the right-hand side in inequality (4-1) becomes

$$
\frac{2 s_{g}-k+\sqrt{k^{2}-4 k s_{g}+4 s_{g}^{2} / n}}{2(n-1)}=n,
$$

and so the result in Theorem 4.2 is optimal.

In fact, in the case $\operatorname{Ric}_{g} \geq k$ and $s_{g}=n k$ - corresponding to the assumption that $g$ is Einstein - the conclusion of Theorem 4.2 is that $\lambda_{1} \leq \frac{n k}{n-1}$. Thus, by the Lichnerowicz-Obata theorem [Lichnerowicz 1958; Obata 1962; Berger et al. 1971], the only Einstein metric with positive constant scalar curvature which is A-superharmonic is the standard metric on the sphere. This fact also shows that the assumption $\operatorname{ker} \mathscr{A} \neq 0$ cannot be removed from Lemma 4.1.

Remark 4.4. Let $\left(M^{n}, g\right)$ be a compact $n$-dimensional Riemannian manifold such that $\operatorname{Ric}_{g} \geq k \geq 0$, where the scalar curvature $s_{g}$ is a positive constant. In addition, suppose that there exists a function $\varphi$ such that $M_{\varphi}^{-}=\{x \in M: \varphi(x)<0\} \neq \varnothing$ and $\mathscr{A} \varphi \geq 0$ on $M_{\varphi}^{-}$. Then, by simply applying Theorem 4.2 to the function $\bar{\varphi}=-\varphi$, we can see that the first nonzero eigenvalue $\lambda_{1}$ of the Laplacian satisfies

$$
\lambda_{1} \leq \frac{2 s_{g}-k+\sqrt{k^{2}-4 k s_{g}+4 s_{g}^{2} / n}}{2(n-1)} .
$$

In particular, if $k=0$, then

$$
\lambda_{1} \leq \frac{s}{n-1}\left(1+\frac{1}{\sqrt{n}}\right)
$$

Finally, we consider the relationship of $v$ to the first nonzero eigenvalue of the Laplace operator. In the case of $v>0$, it follows from Theorem 3.2 that a minimizer $u$ for the functional $E$ satisfies $\mathscr{A} u=v u$. In particular, since $\operatorname{ker} s_{g}^{\prime *}=0$ when $v>0$, we cannot, in general, expect that $s_{g} /(n-1)$ is contained in the spectrum of the Laplace operator.

Theorem 4.5. Let $(M, g)$ be a compact n-dimensional Riemannian manifold such that $\operatorname{Ric}_{g} \geq k \geq 0$ and assume that $v>s_{g}^{2} / n$, where the scalar curvature $s_{g}$ is a positive constant. In addition, suppose that $M_{u}^{+} \neq \varnothing$ for a function u satisfying

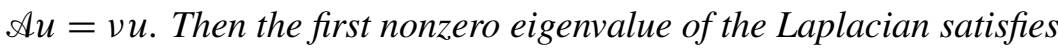

$$
\lambda_{1}(M) \leq \frac{2 s_{g}-k+\sqrt{k^{2}-4 k s_{g}+4 s_{g}^{2} / n+4(n-1) v}}{2(n-1)},
$$

unless $(M, g)$ is Einstein. 
Proof. We shall denote $s_{g}$ by $s$ and $\operatorname{Ric}_{g}=r_{g}$ by $r$. From $\int_{M} u A u=v \int_{M} u^{2}$,

$$
\begin{aligned}
0 & =\int_{M} u A u-v u^{2}=\int_{M}(n-1)(\Delta u)^{2}+2 s u \Delta u+r(d u, d u)+\left(|r|^{2}-v\right) u^{2} \\
& >\int_{M}(n-1)(\Delta u)^{2}+(2 s-k) u \Delta u+\left(\frac{s^{2}}{n}-v\right) u^{2} .
\end{aligned}
$$

The third inequality is strict since $(M, g)$ is not Einstein. We may factor the integrand as follows:

$(n-1)(\Delta u)^{2}+(2 s-k) u \Delta u+\left(\frac{s^{2}}{n}-v\right) u^{2}=((n-1) \Delta u+\alpha u)\left(\Delta u+\frac{\beta}{n-1} u\right)$,

where

$$
\begin{aligned}
& \alpha=\frac{1}{2}\left(2 s-k+\sqrt{k^{2}-4 k s+\frac{4 s^{2}}{n}+4(n-1) v}\right), \\
& \beta=\frac{1}{2}\left(2 s-k-\sqrt{k^{2}-4 k s+\frac{4 s^{2}}{n}+4(n-1) v}\right) .
\end{aligned}
$$

Note that if $v>s^{2} / n$, the radicand is positive for any $k \geq 0$.

The remainder of the proof is similar to that of Theorem 4.2. Hence, if $g$ is not an Einstein metric and $\lambda>\frac{\alpha}{n-1}$, then

$$
0 \geq \int_{M} u A u-v u^{2}>\int_{M}(n-1)(\Delta u)^{2}+(2 s-k) u \Delta u+\left(\frac{s^{2}}{n}-v\right) u^{2} \geq 0,
$$

which is a contradiction.

Theorem 4.6. Let $(M, g)$ be a compact n-dimensional Riemannian manifold such that Ric $_{g} \geq k$ with

$$
0 \leq k \leq 2 s_{g}\left(1-\sqrt{1-\frac{1}{n}-(n-1) \frac{v}{s_{g}^{2}}}\right) .
$$

Suppose that $0<v \leq s_{g}^{2} / n$. In addition, assume that $M_{u}^{+} \neq \varnothing$ for a function $u$ satisfying $A u=v u$. Then the first nonzero eigenvalue $\lambda_{1}$ of the Laplacian satisfies

$$
\lambda_{1} \leq \frac{2 s_{g}-k+\sqrt{k^{2}-4 k s_{g}+4 s_{g}^{2} / n+4(n-1) v}}{2(n-1)},
$$

unless $(M, g)$ is Einstein.

Proof. Note that if $v \leq s_{g}^{2} / n$ and (4-6) is satisfied,

$$
k^{2}-4 k s_{g}+\frac{4 s_{g}^{2}}{n}+4(n-1) v \geq 0 .
$$

The remainder of the proof proceeds in the same manner as that of Theorem 4.5. 


\section{Acknowledgement}

The authors would like to express their gratitude to the referee for several valuable comments, which allowed us to express some arguments more clearly and finely.

\section{References}

[Berger and Ebin 1969] M. Berger and D. Ebin, "Some decompositions of the space of symmetric tensors on a Riemannian manifold", J. Differential Geometry 3 (1969), 379-392. MR 42 \#993 Zbl 0194.53103

[Berger et al. 1971] M. Berger, P. Gauduchon, and E. Mazet, Le spectre d'une variété riemannienne, Lecture Notes in Mathematics 194, Springer, Berlin, 1971. MR 43 \#8025 Zbl 0223.53034

[Besse 1987] A. L. Besse, Einstein manifolds, Ergebnisse der Math. (3) 10, Springer, Berlin, 1987. MR 88f:53087 Zbl 0613.53001

[Bourguignon 1975] J.-P. Bourguignon, "Une stratification de l'espace des structures riemanniennes", Compositio Math. 30 (1975), 1-41. MR 54 \#6189 Zbl 0301.58015

[Fischer and Marsden 1974] A. E. Fischer and J. E. Marsden, "Manifolds of Riemannian metrics with prescribed scalar curvature", Bull. Amer. Math. Soc. 80 (1974), 479-484. MR 49 \#11561 Zbl 0288.53040

[Lichnerowicz 1958] A. Lichnerowicz, Géométrie des groupes de transformations, Travaux et Recherches Mathématiques 3, Dunod, Paris, 1958. MR 23 \#A1329 Zbl 0096.16001

[Obata 1962] M. Obata, "Certain conditions for a Riemannian manifold to be isometric with a sphere", J. Math. Soc. Japan 14 (1962), 333-340. MR 25 \#5479 Zbl 0115.39302

[Struwe 1990] M. Struwe, Variational methods: Applications to nonlinear partial differential equations and Hamiltonian systems, Springer, Berlin, 1990. MR 92b:49002 Zbl 0746.49010

Received December 2, 2011. Revised September 26, 2012.

SEUNGSU HWANG

DEPARTMENT OF MATHEMATiCS

CHUNG-ANG UNIVERSITY

SEOUL 156-756

SOUTH KOREA

seungsu@cau.ac.kr

JEONGWOOK CHANG

DEPARTMENT OF MATHEMATICS EDUCATION

DANKOOK UNIVERSITY

GYEONG-GI 448-701

SOUTH KOREA

jchang@dankook.ac.kr

GABJIN YUN

DEPARTMENT OF MATHEMATICS

MYONG JI UNIVERSITY

GYEONG-GI 449-728

SOUTH KOREA

gabjin@mju.ac.kr 


\title{
PACIFIC JOURNAL OF MATHEMATICS
}

\author{
msp.org/pjm
}

Founded in 1951 by E. F. Beckenbach (1906-1982) and F. Wolf (1904-1989)

\section{EDITORS}

V. S. Varadarajan (Managing Editor)

Department of Mathematics

University of California

Los Angeles, CA 90095-1555

pacific@math.ucla.edu

Paul Balmer

Department of Mathematics

University of California

Los Angeles, CA 90095-1555

balmer@math.ucla.edu

Daryl Cooper

Department of Mathematics

University of California

Santa Barbara, CA 93106-3080 cooper@math.ucsb.edu

Jiang-Hua $\mathrm{Lu}$

Department of Mathematics

The University of Hong Kong

Pokfulam Rd., Hong Kong jhlu@maths.hku.hk
Don Blasius

Department of Mathematics University of California

Los Angeles, CA 90095-1555

blasius@math.ucla.edu

Robert Finn

Department of Mathematics Stanford University

Stanford, CA 94305-2125

finn@math.stanford.edu

Sorin Popa

Department of Mathematics

University of California

Los Angeles, CA 90095-1555

popa@math.ucla.edu

Paul Yang

Department of Mathematics

Princeton University

Princeton NJ 08544-1000

yang@math.princeton.edu

\section{PRODUCTION}

Silvio Levy, Scientific Editor, production@msp.org

\section{SUPPORTING INSTITUTIONS}

ACADEMIA SINICA, TAIPEI

CALIFORNIA INST. OF TECHNOLOGY

INST. DE MATEMÁTICA PURA E APLICADA

KEIO UNIVERSITY

MATH. SCIENCES RESEARCH INSTITUTE

NEW MEXICO STATE UNIV.

OREGON STATE UNIV.

\author{
STANFORD UNIVERSITY \\ UNIV. OF BRITISH COLUMBIA \\ UNIV. OF CALIFORNIA, BERKELEY \\ UNIV. OF CALIFORNIA, DAVIS \\ UNIV. OF CALIFORNIA, LOS ANGELES \\ UNIV. OF CALIFORNIA, RIVERSIDE \\ UNIV. OF CALIFORNIA, SAN DIEGO \\ UNIV. OF CALIF., SANTA BARBARA
}

\author{
Vyjayanthi Chari \\ Department of Mathematics \\ University of California \\ Riverside, CA 92521-0135 \\ chari@math.ucr.edu \\ Kefeng Liu \\ Department of Mathematics \\ University of California \\ Los Angeles, CA 90095-1555 \\ liu@math.ucla.edu \\ Jie Qing \\ Department of Mathematics \\ University of California \\ Santa Cruz, CA 95064 \\ qing@cats.ucsc.edu
}

These supporting institutions contribute to the cost of publication of this Journal, but they are not owners or publishers and have no responsibility for its contents or policies.

See inside back cover or msp.org/pjm for submission instructions.

The subscription price for 2013 is US \$400/year for the electronic version, and \$485/year for print and electronic.

Subscriptions, requests for back issues and changes of subscribers address should be sent to Pacific Journal of Mathematics, P.O. Box 4163, Berkeley, CA 94704-0163, U.S.A. The Pacific Journal of Mathematics is indexed by Mathematical Reviews, Zentralblatt MATH, PASCAL CNRS Index, Referativnyi Zhurnal, Current Mathematical Publications and the Science Citation Index.

The Pacific Journal of Mathematics (ISSN 0030-8730) at the University of California, c/o Department of Mathematics, 798 Evans Hall \#3840, Berkeley, CA 94720-3840, is published monthly except July and August. Periodical rate postage paid at Berkeley, CA 94704, and additional mailing offices. POSTMASTER: send address changes to Pacific Journal of Mathematics, P.O. Box 4163, Berkeley, CA 94704-0163.

PJM peer review and production are managed by EditFLOW ${ }^{\circledR}$ from Mathematical Sciences Publishers.

PUBLISHED BY

mathematical sciences publishers

nonprofit scientific publishing

http://msp.org/

(C) 2013 Mathematical Sciences Publishers 


\section{PACIFIC JOURNAL OF MATHEMATICS}

Volume $261 \quad$ No. $2 \quad$ February 2013

Geography of simply connected nonspin symplectic 4-manifolds with positive signature

Anar Akhmedov, Mark C. Hughes and B. Doug Park

Schur-Horn theorems in $\mathrm{II}_{\infty}$-factors

283

MARTÍN ARGERAMI and PEDRo MASSEY

Classification of positive solutions for an elliptic system with a higher-order

fractional Laplacian

JiNGBo DOU and CHANGZHENG QU

Bound states of asymptotically linear Schrödinger equations with compactly supported potentials

MingWEN FEI and HUICHENG YiN

Type I almost homogeneous manifolds of cohomogeneity one, III

DANIEL GUAN

The subrepresentation theorem for automorphic representations

MARCELA HANZER

Variational characterizations of the total scalar curvature and eigenvalues of the

Laplacian

Seungsu Hwang, JeOngwook Chang and GabJin Yun

Fill-ins of nonnegative scalar curvature, static metrics, and quasi-local mass

JEFFREY L. JAUREGUI

Operator algebras and conjugacy problem for the pseudo-Anosov automorphisms of a surface

\section{IGOR NIKOLAEV}

Connected sums of closed Riemannian manifolds and fourth-order conformal invariants

DAVID RASKE

Ruled minimal surfaces in the three-dimensional Heisenberg group

Heayong Shin, Young Wook Kim, Sung-Eun Koh, Hyung Yong LeE and SEONG-DEOG YANG

$G$-bundles over elliptic curves for non-simply laced Lie groups and configurations of lines in rational surfaces

MANG XU and JIAJIN ZHANG 